\title{
Perfect Anomalous Reflection with a Bipartite Huygens' Metasurface
}

\author{
Alex M. H. Wong and George V. Eleftheriades" \\ The Edward S. Rogers Department of Electrical and Computer Engineering, \\ University of Toronto, Toronto, Canada M5S $3 G 4$
}

(Received 29 September 2017; revised manuscript received 3 December 2017; published 28 February 2018)

\begin{abstract}
In this paper, we propose a new metasurface that is able to reflect a known incoming electromagnetic wave into an arbitrary direction, with perfect power efficiency. This seemingly simple task, which we hereafter call perfect anomalous reflection, is actually highly nontrivial because of the differing wave impedances and complex interference between the incident and reflected waves. Heretofore, proposed metasurfaces that achieve perfect anomalous reflection require complicated, deeply subwavelength and/or multilayer element structures, which allow them to couple to and from leaky and/or evanescent waves. In contrast, we demonstrate that using a bipartite Huygens' metasurface (BHM) - a passive and lossless metasurface with only two cells per period-perfect anomalous reflection can be achieved over a wide angular and frequency range. Through simulations and experiments at $24 \mathrm{GHz}$, we show that a properly designed BHM can anomalously reflect an incident electromagnetic wave from $\theta_{i}=50^{\circ}$ to $\theta_{r}=-22.5^{\circ}$, with perfect power efficiency to within experimental precision.
\end{abstract}

DOI: 10.1103/PhysRevX.8.011036

Subject Areas: Metamaterials, Optics

\section{INTRODUCTION}

The ability to direct the flow of electromagnetic waves at will has captured the fascination of scientists and engineers. Potential applications include scientific and medical imaging, information and communication technology, and energy harvesting, among many others. Recent decades have witnessed the emergence of metamaterials and metasurfaces that provide designer-defined properties to direct electromagnetic waves in an arbitrary manner [1-23]. Specifically, a phase-gradient metasurface has been heavily investigated as a ubiquitous tool to control the reflection and transmission of a known incident wave [15,21-23]. More recently, the Huygens' metasurface-a surface which provides both electric and magnetic responses to an incoming EM wave-has garnered attention as a metasurface with ultimate wave manipulation capabilities [18-20,24-26]. For instance, it has been shown that total transmission and redirection of an incident wave is possible with a passive bianisotropic Huygens' metasurface, implementable on three closely-spaced layers of reactive elements [27].

The problem of arbitrarily reflecting an electromagnetic wave, however, has proven surprisingly tricky. Prior to

\footnotetext{
*gelefth@ece.utoronto.ca
}

Published by the American Physical Society under the terms of the Creative Commons Attribution 4.0 International license. Further distribution of this work must maintain attribution to the author(s) and the published article's title, journal citation, and DOI. works on metasurfaces, researchers studying blazed gratings-gratings that perform efficient retroreflectionfound that some thick gratings could redirect a wave into directions other than retroreflection with very high efficiency. These so-called "off-Bragg" gratings were numerically demonstrated for small to moderate separation angles [28-30]. A theoretical investigation [31] found, from the perspective of plane-wave diffraction, that perfect anomalous reflection was possible given the successful achievement of two angular parameters. The extent to which these parameters can be tuned remained unclear, but the authors of [31] further showed that for specific incidence and diffracted angles, the required parameters can be achieved by tuning the groove depth of a rectangular groove grating. However, recent works on metasurfaces [32-34] considered the electromagnetic waves at an impedance boundary and showed that, in order to steer an incident wave into an "anomalous" direction with perfect efficiency, one needs a reflection surface that is lossy (absorbs the incident wave) in some areas and active (reradiates more power than in the incident wave) in other areas. This finding raises questions on how a passive structure, like a grating, can achieve perfect anomalous reflection. Further, this explains why phase-gradient metasurfaces [15] and some Huygens' metasurfaces [18,19] failed to achieve perfect anomalous reflection, but instead generated undesirable spurious reflection and/or transmission components.

Several alternatives have thus been proposed to achieve anomalous reflection. The authors of Ref. [20] first reported the reduction of spurious reflection in a metasurface with an appropriately designed loss profile. Later, the 
authors of Refs. [32,33] showed that for the plane-wave reflection case, one can completely eliminate spurious components by including loss in a passive metasurface. This allows one to achieve anomalous reflection at an efficiency limited by the impedance mismatch, given by

$$
\left(\frac{P_{\text {out }}}{P_{\text {in }}}\right)_{\text {max }}=\min \left(\frac{\cos \theta_{r}}{\cos \theta_{i}}, \frac{\cos \theta_{i}}{\cos \theta_{r}}\right) .
$$

Alternatively, more power can be anomalously transmitted if one also allows some of the incident wave to be scattered into other directions. However, in both of these cases, the impedance mismatch makes it impossible to efficiently redirect a wave to or from near-grazing angles. More recently, the authors of Refs. [34-36] proposed specialized metasurfaces that redistribute power along the surface using auxiliary evanescent waves [34] or leaky waves [35,36], to achieve the aforementioned absorptive and radiation regions using a passive and lossless metasurface. However, in these cases, the respective authors have not suggested a straightforward process through which one can design physical metasurface structures capable of achieving the aforementioned power transfer or auxiliary wave generation. Furthermore, the proposed metasurfaces are conceptually complex, in that they require very intricate coupling mechanisms, deeply subwavelength element dimensions, and intricate metallization patterns and/or multilayer bianisotropic metasurface elements [34-36].

In this paper, we report a very simple metasurface that successfully facilitates the aforementioned power redistribution and achieves perfect anomalous reflection, without any explicit involvement of surface or leaky waves. We show that maximally discretized metasurfaces-in some cases, with as few as two simple elements per period-can be built to reflect an electromagnetic wave from a given input direction to a desired output direction. As an example, we demonstrate perfect anomalous reflection from an incident direction $\theta_{i}=50^{\circ}$ to a reflection direction $\theta_{r}=$ $-22.5^{\circ}$ using a bipartite Huygens' metasurface (BHM) - a Huygens' metasurface with only two elements per period. Experimental results corroborate with simulation to show near-perfect power transfer from the incident to the desired reflection direction and show that efficient reflection of greater than $90 \%$ power transfer from the incident to the redirected wave can be achieved over a wide bandwidth of $25.7 \%$. Our results demonstrate that such perfect anomalous reflection can be achieved without resorting to intricate, active, or lossy metasurface profiles, and that this realization leads to superior metasurfaces capable of broadband performance. Furthermore, the development in this paper reconciles current works on metasurfaces with previous results in dielectric and metallic gratings: It offers a plausible explanation for the fundamental electromagnetics behind previous studies of near-perfect anomalous reflection from off-Bragg gratings.

\section{RESULTS}

\section{A. Aggressive metasurface discretization}

We begin by describing our theoretical results regarding the discretization of a general periodic metasurface, which includes but is not limited to a perfect anomalous reflector. The common method for metasurface design is to design a continuous impedance-admittance surface that satisfies the interface boundary condition between a given and a desired electromagnetic wave. Thereafter, the surface is finely discretized and implemented using metasurface elements with corresponding electromagnetic properties. Typical metasurface discretizations range from 8 to 12 cells per wavelength (or, in some cases, per period) $[18,26,37]$. These discretizations are usually verified to be of sufficiently fine granularity by yielding results that approximate the theoretical or simulated performance of the continuous counterpart. However, we propose to investigate and implement a metasurface that is as aggressively discretized as possible in regards to the number of elements per period. We shall show that an aggressively discretized metasurface will lead to simple, robust, and broadband metasurface implementation. Also, we shall show that the aggressively discretized metasurface has a counterintuitive effect of allowing possibilities unattainable by its continuous counterpart, which, in the case of this paper, enables it to achieve perfect anomalous reflection without explicitly involving a transfer of power through surface or leaky waves.

We employ a diffraction-based perspective to investigate sufficient discretization of a periodic metasurface [38]. Figure 1(a) shows the $k$-space operation of a metasurface

(a)

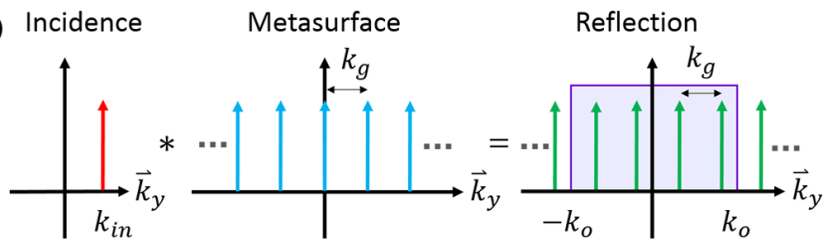

(b)

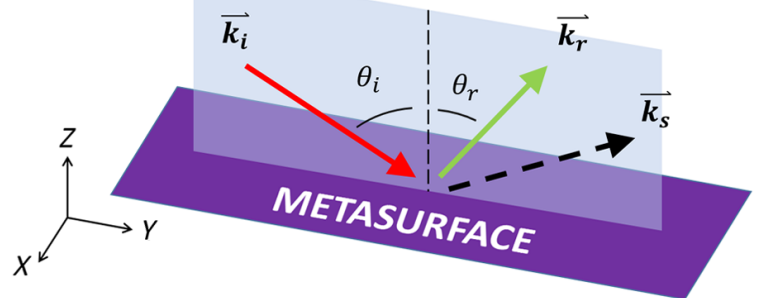

FIG. 1. Perfect anomalous reflection with a discretized periodic metasurface. (a) A diagram describing how a periodic metasurface generates diffraction modes. Arrows indicate the existence (not the amplitudes) of diffraction modes; the purple box denotes the regime of propagation waves. $y$ denotes the direction of variation along the metasurface. (b) A schematic diagram of a perfect anomalous reflector, which redirects the incident wave $\left(\mathbf{k}_{\mathbf{i}}\right)$ into an anomalous direction $\left(\mathbf{k}_{\mathbf{r}}\right)$ and totally suppresses specular reflection $\left(\mathbf{k}_{\mathrm{s}}\right)$. 
with period $\Lambda_{g}$ and wave number $k_{g}=2 \pi / \Lambda_{g}$. Upon the incidence of a plane wave, the periodic metasurface reflects multiple diffraction modes depicted by the location of the arrows. The amplitude and phase of the diffraction modes are dependent on the reflective properties of the metasurface. It is instructive to note that, while an infinite number of diffraction modes exist in $k$-space, only a finite number of diffraction modes fall within the propagation range of $k_{y} \in\left[-k_{0}, k_{0}\right]$. These represent plane waves that scatter into the far field, with the propagation angle given by

$$
\sin \theta_{r}=\frac{k_{y}}{k_{0}} \quad \text { for }\left|k_{y}\right| \leq k_{0} .
$$

The other diffraction modes represent evanescent waves that remain within the near field of the metasurface, but do not contribute to the far-field reflection pattern. The number of outgoing diffraction modes is given by

$$
N=1+\left\lfloor\frac{k_{0}-k_{i}}{k_{g}}\right\rfloor+\left\lfloor\frac{k_{0}+k_{i}}{k_{g}}\right\rfloor .
$$

Here, $\lfloor\cdot\rfloor$ indicates the floor (round down) operation. We show in the Supplemental Material that a metasurface with $N$ independent degrees of freedom is sufficient for tuning the reflection dynamics into each propagation diffraction mode [39]. Further, we show that these $N$ degrees of freedom can be met by regenerating the electromagnetic fields at $N$ sample points, equidistant along the length of the period, using an array of $N$ elements.

It is of interest to note that, for a class of metasurfaces with sufficiently large spatial frequency such that $k_{g} \in\left[k_{0}, 2 k_{0}\right)$, there exists only two propagating diffraction modes. This condition is satisfied by many metasurfaces: for example, in retroreflection metasurfaces with $\left|\theta_{i}\right|=$ $\left|\theta_{r}\right| \geq 19.5^{\circ}[40-42]$. We shall show in the next subsection that this also is the case for many perfect anomalous reflectors. For these cases, the metasurface can be aggressively discretized to having only two elements per grating period [38]. We name the resultant surface a bipartite Huygens' metasurface (BHM). In a previous work, we designed a BHM to perform near-grazing angle retroreflection [42]. We shall proceed to show that a properly designed BHM can also perform perfect anomalous reflection across a wide range of frequencies.

\section{B. Perfect anomalous reflector: Design and simulation}

We now concentrate our discussion on a perfect anomalous reflection metasurface. A schematic of a perfect anomalous reflector is shown in Fig. 1(b). The surface accepts an incident wave at $\theta_{i}$ and anomalously reflects it at an anomalous reflection angle $\theta_{r}$. Specular reflection $\left(\mathbf{k}_{\mathbf{s}}\right)$ is totally suppressed. To construct an aggressively discretized perfect anomalous reflection metasurface, one can choose the metasurface period such that $k_{g}=k_{r}-k_{i}=$ $k_{0}\left(\sin \theta_{r}-\sin \theta_{i}\right)$. Substituting this into (3) yields

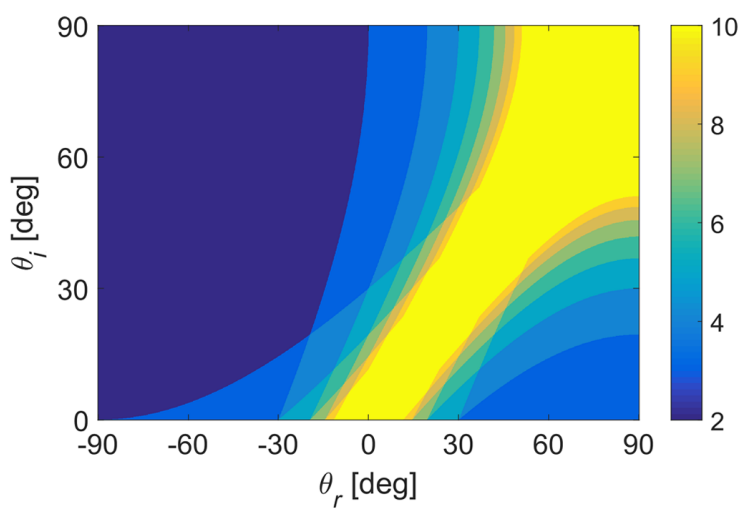

FIG. 2. A plot showing $N$ as a function of $\left(\theta_{i}, \theta_{r}\right)$.

$$
N=1+\left\lfloor\frac{\left(1-\sin \theta_{i}\right)}{\left|\sin \theta_{r}-\sin \theta_{i}\right|}\right\rfloor+\left\lfloor\frac{\left(1+\sin \theta_{i}\right)}{\left|\sin \theta_{r}-\sin \theta_{i}\right|}\right\rfloor .
$$

Figure 2 plots this relationship for $\theta_{i} \in\left[0^{\circ}, 90^{\circ}\right]$ and $\theta_{r} \in\left[-90^{\circ}, 90^{\circ}\right]$. The sizable region shaded in dark blue $(N=2)$ on the left side of the plot indicates the region for which there exists only two propagating diffraction modes. Hence, a bipartite Huygens' metasurface suffices to perfectly redirect the incident power from the incident to the anomalous reflection angle. Hence, for the remaining regions $N>2$, more metasurface elements are needed for every period. The yellow region $(N \geq 10)$ denotes sets of $\left(\theta_{i}, \theta_{r}\right)$ for which many (ten or more) elements are needed. However, this region also represents "weak" anomalous reflection, in the sense that the reflection angle is only slightly deviated from the incident angle $\left(\theta_{r} \approx \theta_{i}\right)$. Therefore, the period is also correspondingly large, which allows the elements to be spaciously spaced despite the need for many elements within one period.

As a design example demonstrating the bipartite Huygens' metasurface, we design our metasurface for the microwave regime at a frequency of $24 \mathrm{GHz}$. We construct the metasurface on a printed circuit board (PCB) with $1.575-\mathrm{mm}$ thickness and a permittivity of $\varepsilon_{r}=2.2$. We choose as the metasurface element the ground-backed dipole, which, as we have shown in previous works [20,38,42], can operate as an efficient Huygens' metasurface component. This also exhibits various desirable properties such as low loss, effective phase tuning, and a reasonably large tolerance to bandwidth and angular variations. A schematic of the unit cell is shown in Fig. 3(a). We seek to design a metasurface that performs perfect anomalous reflection to convert a TE wave from an incident angle of $50^{\circ}$ to an anomalously reflected angle of $-22.5^{\circ}$. A substitution into (4) shows that there are only two propagation modes for a metasurface with this periodicity. Hence, a bipartite Huygens' metasurface can achieve perfect anomalous reflection into the required direction.

We describe our design process and report full-wave electromagnetic simulation results using the commercial 


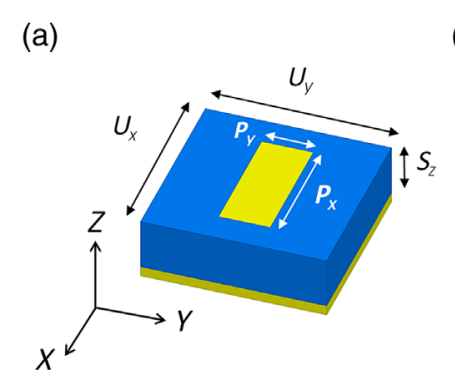

(b)

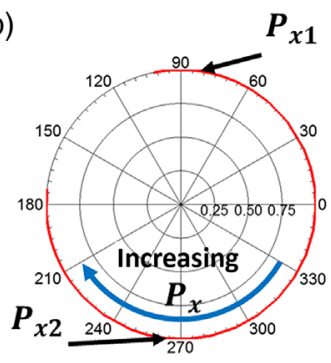

(c)

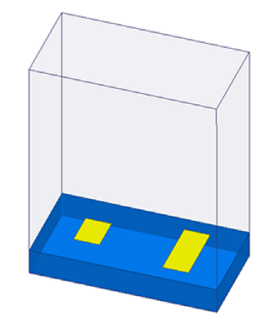

(d)

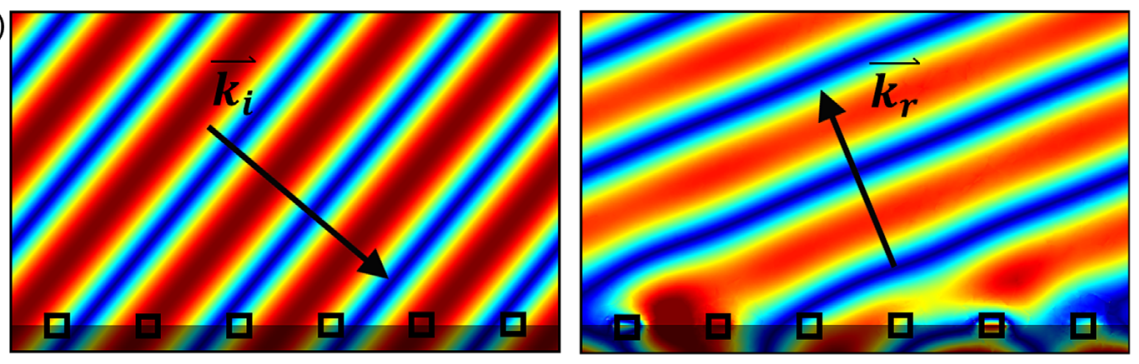

FIG. 3. Design and simulation of a perfect anomalous reflection metasurface. (a) A metasurface element cell. $U_{x}=U_{y}=\Lambda / 2$, $S_{z}=1.575 \mathrm{~mm}, P_{y}=1.5 \mathrm{~mm}$, and $P_{x}$ is swept to generate the phase shift. (b) The reflection coefficient as $P_{x}$ is varied. The chosen operation points $\left(P_{x 1}, P_{x 2}\right)$ are labeled. (c) A schematic of one period of the bipartite Huygens' metasurface. (d) $|\mathbf{E}(\mathbf{r}, t)|$ in the plane of incidence (the $y z$ plane), showing the electric field magnitude of the incident (left) and the scattered (right) waves. In both cases, the darkened strip denotes the metasurface, and black squares highlight the location of the dipoles.

simulator Ansys HFSS. We first characterize the reflection properties of a single element when placed in an infinitely periodic array in the $x$ and $y$ directions. This is achieved by a full-wave Floquet (periodic) simulation. An electromagnetic plane wave, with electric field pointing in the $x$ direction, impinges the element at $\theta_{i}=50^{\circ}$, and specular reflection is measured. Figure 3 (b) shows the variation in specular reflection as we vary the dipole length $P_{x}$. We observe that we achieve a near-unity reflection magnitude throughout the sweep, while the reflection phase differs by about $180^{\circ}$ at the operation points $P_{x 1}=2.0 \mathrm{~mm}$ and $P_{x 2}=3.4 \mathrm{~mm}$. As in Ref. [42], we combine elements that exhibit a $180^{\circ}$ difference in phase behavior to suppress specular reflection and thereby efficiently redirect an incoming wave toward the desired anomalous reflection.

Hence, we combine the aforementioned elements to form the BHM. When these metasurface elements are placed adjacent to each other, their mutual coupling dynamics cause slight deviations in their reflection properties, which in turn degrade the suppression of specular reflection. To account for this, we sweep $P_{x 2}$ from 3.4 to $3.8 \mathrm{~mm}$ in this new environment and find that, at $P_{x 2}=3.6 \mathrm{~mm}$, we reestablish destructive interference in the specular direction and thereby optimize power transfer to the anomalous direction. The resultant Floquet simulation shows that this BHM transfers $99.98 \%$ of the incident power from $\theta_{i}=50^{\circ}$ to $\theta_{r}=-22.5^{\circ} ; 0.02 \%$ of the power remains as specular reflection. This demonstrates "perfect" anomalous reflection to within the accuracy of the simulation and certainly exceeds the power transfer efficiency as dictated by the impedance mismatch relationship (1).
Figure 3(d) shows the incident and scattered waves obtained from the simulation; animations showing the phase progression of both waves are available in the Supplemental Material that accompanies this paper [43]. Both the figure and the animations show that the incident wave is reflected anomalously in a near-perfect manner. In accordance to electromagnetic theory, in a scenario of perfect power transfer from the incident wave into anomalous reflection, the reflected wave features an electric field that differs in amplitude from the incident wave:

$$
\frac{\left|E_{x, r}\right|}{\left|E_{x, i}\right|}=\sqrt{\frac{\cos \theta_{i}}{\cos \theta_{r}}}=0.8341 \quad \text { (for our case). }
$$

Notwithstanding this lower field amplitude, perfect power transfer is achieved because the anomalously reflected wave carries power more efficiently in the $z$ direction.

\section{Power flow analysis}

Figure 4 examines the power flow dynamics of the BHM. The top panel of the figure shows the geometry of the metasurface, while the middle three panels show the $z-$ directed Poynting vector $\operatorname{Re}\left\{S_{z}\right\}$ (electromagnetic power flow) across three planes, at distances $z=\lambda / 25, \lambda / 6$, and $\lambda / 2$ above the metasurface. $\operatorname{Re}\left\{S_{z}\right\}>0$ implies that power is radiated by the metasurface; conversely, $\operatorname{Re}\left\{S_{z}\right\}<0$ implies that power is absorbed. In close proximity to the metasurface $(z=\lambda / 25)$, we see that the power flow forms an intricate pattern due to the reactive near field. However, 


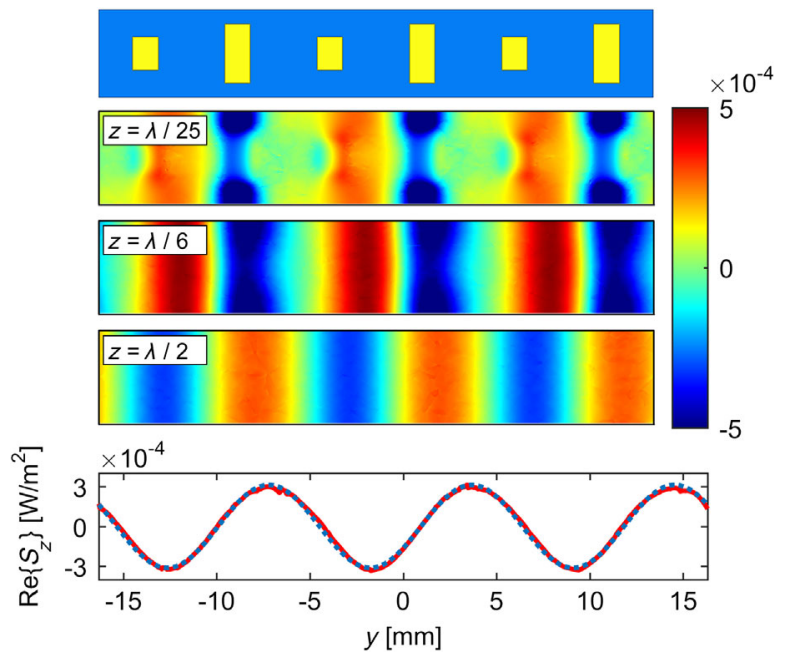

FIG. 4. Power flow analysis. The top panel shows the metasurface geometry across three spatial periods. The second to fourth panels plot power flow $\left(\operatorname{Re}\left\{S_{z}\right\}\right)$ across the planes at designated distances above the metasurface. The bottom panel compares $\operatorname{Re}\left\{S_{z}(x=0, z=\lambda / 2)\right\}$ (solid red curve) against theory (dashed blue curve), as calculated by (6).

as $z$ increases, the evanescent field diminishes and $\operatorname{Re}\left\{S_{z}\right\}$ approaches a sinusoid. The bottom panel plots the variation at $x=0$ and $z=\lambda / 2$ (solid red curve). It shows that this variation very well approximates the theoretical sinusoidal variation (dotted blue curve) of a perfect anomalous reflection metasurface [32,33],

$\operatorname{Re}\left\{S_{z}\right\}=\frac{\left|E_{x, i}\right|^{2}}{2 \eta_{0}} \sqrt{\frac{\cos \theta_{i}}{\cos \theta_{r}}}\left(\cos \theta_{r}-\cos \theta_{i}\right) \cos \left(k_{g} y-\phi\right)$,

where $\eta_{0}$ is the free-space wave impedance and $\phi$ is a constant reflection phase offset. It was previously noted that perfect anomalous reflection required a redistribution of power, such that half the surface is active $\left(\operatorname{Re}\left\{S_{z}\right\}>0\right)$ and the other half is lossy $\left(\operatorname{Re}\left\{S_{z}\right\}<0\right)$. We have hereby shown that, notwithstanding its apparent simplicity, the BHM successfully accomplishes this necessary redistribution of power through aggressive discretization and the consideration of diffraction modes. We find that this process involves evanescent waves as in [34], but in an implicit manner that simplifies rather than complicates the task of metasurface design and construction.

\section{Finite surface and bandwidth analysis}

We proceed to simulate the reflection properties of the metasurface when it is truncated to a finite size of 78 cells $(413.4 \mathrm{~mm})$ in the $y$ direction. Figure 5 (top panel) shows the reflection characteristics under a $24-\mathrm{GHz}$ plane wave illumination at the designed incident angle. Specular

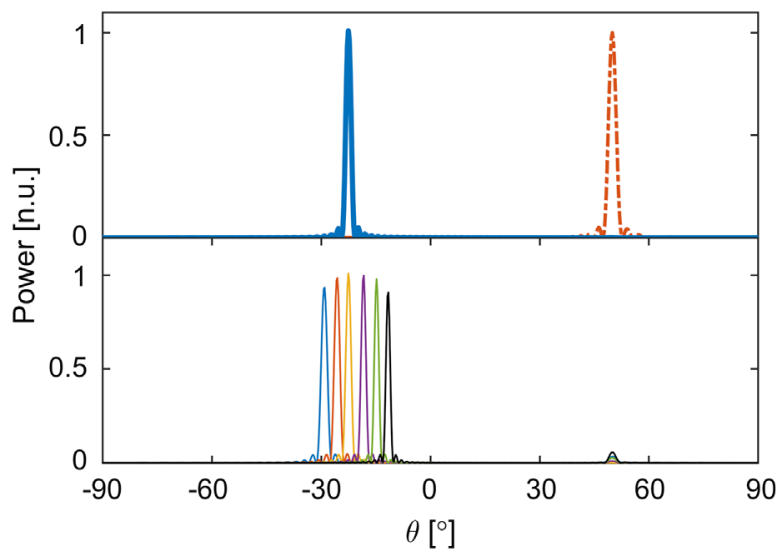

FIG. 5. Simulated scattering of a finite metasurface. The top panel compares the normalized scattering magnitude of the BHM (solid blue curve) with that of a metallic plate of the same size (dashed-dotted red curve). The bottom panel shows the BHM's scattering magnitude over a range of frequencies. Displayed frequencies (from left to right) are 22, 23, 24, 25.5, 27, and $28.5 \mathrm{GHz}$.

reflection is suppressed to $30 \mathrm{~dB}$ (1000 times) below the anomalous reflection power; hence, most of the power is anomalously reflected to the desired direction of $\theta_{r}=-22.5^{\circ}$. To obtain a measurement of power efficiency, the peak of the scattered wave is normalized to the geometric mean of simulated specular reflections for $\theta_{i 1}=$ $50^{\circ}$ and $\theta_{i 2}=22.5^{\circ}$ for a perfect conductor of the same size as the metasurface. (We refer the interest reader to the Supplemental Material for a detailed explanation of our normalization procedure [39].) Hence, a peak magnitude of $0.03 \mathrm{~dB}$ (or $100.7 \%$ ) conveys that the finite-sized metasurface has achieved perfect anomalous reflection to within the accuracy of the simulation.

To illustrate the broadband performance of the metasurface, Fig. 5 (bottom panel) shows the reflection characteristics for a range of frequencies from 22 to $28.5 \mathrm{GHz}$. Because of the nonresonant nature of the metasurface elements, the specular reflection remains significantly suppressed throughout this bandwidth, thus enabling the metasurface to achieve anomalous reflection at a very high ( $\geq 90 \%$ ) efficiency over this bandwidth. Beam squintingthe variation in beam angle with respect to frequency [44] is observed in accordance with antenna array theory. While perfect anomalous reflection is demonstrated at the design frequency, a high-efficiency anomalous reflection of over 90\% and significant improvement over the limitation of the impedance mismatch is also achieved over this very large fractional bandwidth of $25.7 \%$.

\section{E. Experimental demonstration}

We fabricated and experimentally measured anomalous reflection from the bipartite Huygens' metasurface. We 

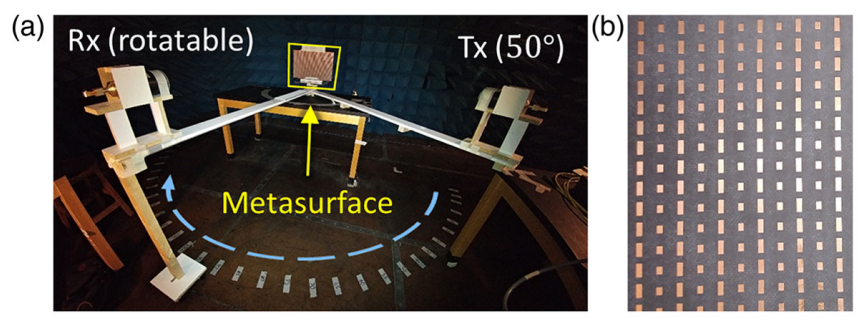

(c)

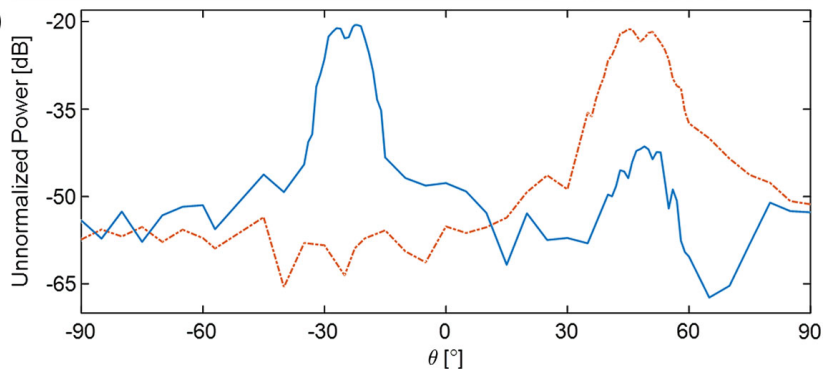

FIG. 6. Experimental demonstration. (a) A photo of the experimental apparatus, showing the transmit (Tx) and receive (Rx) antennas and the test surface platform. (b) A close-up of the BHM. (c) Experimentally measured bistatic RCS for the BHM (solid blue curve) compared against that for a metallic plate of the same size (dashed-dotted red curve).

fabricated the BHM on a Rogers RT/Duroid 5880 laminate board, with $1.575-\mathrm{mm}$ thickness and plated with $1 \mathrm{oz}$ $(35.6 \mu \mathrm{m})$ of copper on both sides. The substrate has a permittivity of $\varepsilon_{r}=2.2$ (as in the simulation) and a low dissipation factor of $\tan \delta \in[0.0004,0.0009]$. Figures 6(a) and $6(\mathrm{~b})$ show the fabricated metasurface and the experimental apparatus. A K-band lens antenna emits a vertically $(x)$ polarized Gaussian beam at $\theta_{i}=50^{\circ}$, about $1.5 \mathrm{~m}$ from the test surface. A second lens antenna receives the scattered signal and is swept across the $y z$ plane to measure the test surface's angular scattering profile. To calibrate the system, we first measure the specular reflection strengths of an aluminum test plate with the same size as the bipartite Huygens' metasurface. We find that the powers reflected from the aluminum plate at $\theta_{i 1}=50^{\circ}$ and $\theta_{i 2}=22.5^{\circ}$ differ by about $0.5 \mathrm{~dB}$ - which is within the expected error of the experimental apparatus. This implies that the incident Gaussian beam is concentrated at the center of the test surface, such that the power loss due to spillover was negligible. Hence, the strength of the anomalous reflection can be found through direct comparison to specular reflection strength for $\theta_{i}=50^{\circ}$. Figure 6(c) compares the scattering pattern of the BHM (solid blue curve) to that of the metallic test plate (dashed-dotted red curve). We observe that the angular spread of the Gaussian beam broadens the reflection features (both by the metallic plate and by the BHM) to a 3-dB (half-power) width of around $10^{\circ}$. Notwithstanding, it is clear that the anomalous reflection surface suppresses specular reflection by about $20 \mathrm{~dB}$ and achieves perfect anomalous reflection to within the sensitivity of the experimental apparatus.

\section{CONCLUSION}

In this paper we have shown that perfect anomalous reflection is possible with an aggressively discretized metasurface. In particular, we demonstrated that a bipartite Huygens' metasurface, built from ground-backed electric dipoles, can steer an incoming electromagnetic wave at $\theta_{i}=50^{\circ}$ to a reflection angle $\theta_{r}=-22.5^{\circ}$, with perfect efficiency to within the tolerance of full-wave simulation and experimental measurement. In a departure from previous proposals, the proposed BHM is surprisingly simple in its single-layer structure and large and simple element sizes, which bodes well for the BHM's practical fabrication even at higher frequencies, such as mm wave, terahertz, and beyond. Further, we demonstrate that because of the nonresonant nature of the elements involved, the reported BHM attains wideband performance: Though the BHM was designed for operation at $24 \mathrm{GHz}$, anomalous reflection with greater than $90 \%$ efficiency was demonstrated from 22 to $28.5 \mathrm{GHz}$. Examining the power flow normal to the metasurface reveals that the discretized metasurface implicitly invokes the necessary evanescent waves that facilitate power redistribution from one part of the metasurface to another, as found necessary in Refs. [32,33]. This implicit involvement of evanescent waves may also have been the operation mechanism through which traditional anomalous reflection gratings achieved the power redistribution along the reflection surface. In this perspective, the present work reconciles earlier observations of seemingly efficient anomalous reflection in gratings with recent developments in electromagnetic metasurfaces.

It may well be noted that the BHM employs a simple structure of a ground-backed dipole array, which in some ways resembles traditional reflectarrays [45] and similarly structured metasurfaces, such as the gradient metasurface $[22,23]$ and the leaky-wave-based perfect anomalous reflection metasurface [35]. Despite this similar structure, the BHM possesses marked differences from the aforementioned reflection structures. Firstly, the BHM features elements less than half a wavelength apart, which distinguishes it from standard reflectarray antennas. Secondly, while we have designed the BHM in consideration of the reflection coefficient (in similitude to reflectarrays and gradient metasurfaces), this design procedure is supported by an analysis on electromagnetic fields that shows that, though simple, this method leads to a cancellation of all spuriously reflected waves. This is not true in general for phase-based metasurfaces, as is shown by recent works $[18,19,46]$ and discussed in the Introduction of this paper. Finally, the aggressively discretized Huygens' metasurface proposed in this paper features relatively large element separations, which allow metasurface elements to (i) have lower quality factors and (ii) become less susceptible to mutual coupling effects. This ultimately relaxes the fabrication tolerance for the metasurface, thereby facilitating low-cost fabrication and robust implementation, even at high operation frequencies. 
The combination of these features and the demonstration of highly efficient $(\geq 90 \%)$ anomalous reflection over a broad bandwidth $(25.7 \%)$ make the BHM a unique and attractive approach for designing a wide range of practical anomalous reflection metasurfaces.

\section{ACKNOWLEDGMENTS}

This works was sponsored by the Natural Sciences and Engineering Research Council of Canada (NSERC).

Note added.-Recently, a work [47] on "metagratings" has come to our attention, which also takes the perspective of diffraction modes to achieve high-efficiency wavefront steering. While the aforementioned work numerically investigated wavefront steering using complex single grating elements, the present paper investigates, through both full-wave simulation and experiment, wavefront design with multiple (two or more) simple elements, distributed within a single period of the metasurface. Further, in this present work the usage of nonresonant metasurface elements has led to the experimental demonstration of broadband anomalous reflection beyond what has been reported by previous works.

[1] V. G. Veselago, The Electrodynamics of Substances with Simultaneously Negative Values of $\epsilon$ and $\mu$, Sov. Phys. Usp. 10, 509 (1968).

[2] J. B. Pendry, Negative Refraction Makes a Perfect Lens, Phys. Rev. Lett. 85, 3966 (2000).

[3] R. A. Shelby, D. R. Smith, and S. Schultz, Experimental Verification of a Negative Index of Refraction, Science 292, 77 (2001).

[4] G. V. Eleftheriades, A. K. Iyer, and P. C. Kremer, Planar Negative Refractive Index Media Using Periodically L-C Loaded Transmission Lines, IEEE Trans. Microwave Theory Tech. 50, 2702 (2002).

[5] N. Fang, H. Lee, C. Sun, and X. Zhang, Sub-DiffractionLimited Optical Imaging with a Silver Superlens, Science 308, 534 (2005).

[6] A. Alù and N. Engheta, Achieving Transparency with Plasmonic and Metamaterial Coatings, Phys. Rev. E 72, 016623 (2005).

[7] J. B. Pendry, D. Schurig, and D. R. Smith, Controlling Electromagnetic Fields, Science 312, 1780 (2006).

[8] Z. Jacob, L. V. Alekseyev, and E. Narimanov, Optical Hyperlens: Far-Field Imaging beyond the Diffraction Limit, Opt. Express 14, 8247 (2006).

[9] M. Silveirinha and N. Engheta, Tunneling of Electromagnetic Energy through Subwavelength Channels and Bends Using E-Near-Zero Materials, Phys. Rev. Lett. 97, 157403 (2006).

[10] I. I. Smolyaninov, Y. J. Hung, and C. C. Davis, Magnifying Superlens in the Visible Frequency Range, Science 315, 1699 (2007).
[11] N. I. Landy, S. Sajuyigbe, J. J. Mock, D. R. Smith, and W. J. Padilla, Perfect Metamaterial Absorber, Phys. Rev. Lett. 100, 207402 (2008).

[12] M. Gharghi, C. Gladden, T. Zentgraf, Y. Liu, X. Yin, J. Valentine, and X. Zhang, A Carpet Cloak for Visible Light, Nano Lett. 11, 2825 (2011).

[13] D. Sievenpiper, L. Zhang, R. F. J. Broas, N. G. Alexopolous, and E. Yablonovitch, High-Impedance Electromagnetic Surfaces with a Forbidden Frequency Band, IEEE Trans. Microwave Theory Tech. 47, 2059 (1999).

[14] C. L. Holloway, M. A. Mohamed, E. F. Kuester, and A. Dienstfrey, Reflection and Transmission Properties of a Metafilm: With an Application to Controllable Surface Composed of Resonant Particles, IEEE Trans. Electromagn. Compat. 47, 853 (2005).

[15] N. Yu, P. Genevet, M. A. Kats, F. Aieta, J. Tetienne, F. Capasso, and Z. Gaburro, Light Propagation with Phase Discontinuities: Generalized Laws of Reflection and Refraction, Science 334, 333 (2011).

[16] S. Maci, G. Minatti, M. Casaletti, and M. Bosiljevac, Metasurfing: Addressing Waves on Impenetrable Metasurfaces, IEEE Antennas Wireless Propag. Lett. 10, 1499 (2011).

[17] C. L. Holloway, E. F. Kuester, J. A. Gordon, J. O'Hara, J. Booth, and D. R. Smith, An Overview of the Theory and Applications of Metasurfaces: The Two-Dimensional Equivalents of Metamaterials, IEEE Antennas Propag. Mag. 54, 10 (2012).

[18] C. Pfeiffer and A. Grbic, Metamaterial Huygens' Surfaces: Tailoring Wave Fronts with Reflectionless Sheets, Phys. Rev. Lett. 110, 197401 (2013).

[19] M. Selvanayagam and G. V. Eleftheriades, Discontinuous Electromagnetic Fields Using Orthogonal Electric and Magnetic Currents for Wavefront Manipulation, Opt. Express 21, 14409 (2013).

[20] M. Kim, A. M. H. Wong, and G. V. Eleftheriades, Optical Huygens' Metasurfaces with Independent Control of the Magnitude and Phase of the Local Reflection Coefficients, Phys. Rev. X 4, 041042 (2014).

[21] D. Lin, P. Fan, E. Hasman, and M. L. Brongersma, Dielectric Gradient Metasurface Optical Elements, Science 345, 298 (2014).

[22] S. Sun, Q. He, S. Xiao, Q. Xu, X. Li, and L. Zhou, GradientIndex Meta-Surfaces as a Bridge Linking Propagating Waves and Surface Waves, Nat. Mater. 11, 426 (2012).

[23] S. Sun, K.-Y. Yang, C.-H. Wang, T.-K. Juan, W. T. Chen, C. Y. Liao, Q. He, S. Xiao, W.-T. Kung, G.-Y. Guo, L. Zhou, and D.P. Tsai, High-Efficiency Broadband Anomalous Reflection by Gradient Meta-Surfaces, Nano Lett. 12, 6223 (2012).

[24] J. P. S. Wong, M. Selvanayagam, and G. V. Eleftheriades, Design of Unit Cells and Demonstration of Methods for Synthesizing Huygens Metasurfaces, Photonics Nanostruct. Fundam. Appl. 12, 360 (2014).

[25] M. Selvanayagam and G. V. Eleftheriades, Polarization Control Using Tensor Huygens Surfaces, IEEE Trans. Antennas Propag. 62, 6155 (2014).

[26] A. Epstein, J. P. S. Wong, and G. V. Eleftheriades, CavityExcited Huygens' Metasurface Antennas for Near-Unity Aperture Illumination Efficiency from Arbitrarily Large Apertures, Nat. Commun. 7, 10360 (2016). 
[27] J. P. S. Wong, A. Epstein, and G. V. Eleftheriades, Reflectionless Wide-Angle Refracting Metasurfaces, IEEE Antennas Propag. Lett. 15, 1293 (2016).

[28] E. V. Jull and N.C. Beaulieu, in IEEE Antennas and Propagation Society International Symposium Digest (IEEE, Quebec, 1980).

[29] Y. K. Cho, J. W. Ra, U. H. Cho, and J. I. Lee, Off-Bragg TE Blazing of a Periodic Strip Grating on a Grounded Dielectric, Electron. Lett. 33, 1446 (1997).

[30] W. Chen, N. C. Beaulieu, D. G. Michelson, and E. V. Jull, Off-Bragg Blazed Rectangular Groove Gratings for High Diffraction Efficiency Devices, IEEE Trans. Antennas Propag. 61, 2342 (2013).

[31] D. Maystre and M. Cadilhac, A Phenomenological Theory for Gratings: Perfect Blazing for Polarized Light in Nonzero Deviation Mounting, Radio Sci. 16, 1003 (1981).

[32] V.S. Asadchy, M. Albooyeh, S. N. Tcvetkova, A. Díaz-Rubio, Y. Ra'di, and S. A. Tretyakov, Perfect Control of Reflection and Refraction Using Spatially Dispersive Metasurfaces, Phys. Rev. B 94, 075142 (2016).

[33] N. M. Estakhri and A. Alù, Wave-Front Transformation with Gradient Metasurfaces, Phys. Rev. X 6, 041008 (2016).

[34] A. Epstein and G. V. Eleftheriades, Synthesis of Passive Lossless Metasurfaces Using Auxiliary Fields for Reflectionless Beam Splitting and Perfect Reflection, Phys. Rev. Lett. 117, 256103 (2016).

[35] A. Díaz-Rubio, V. Asadchy, A. Elsakka, and S. Tretyakov, From the Generalized Reflection Law to the Realization of Perfect Anomalous Reflectors, Sci. Adv. 3, e1602714 (2017).

[36] V. S. Asadchy, A. Wickberg, A. Díaz-Rubio, and M. Wegener, Eliminating Scattering Loss in Anomalously Reflecting Optical Metasurfaces, ACS Photonics 4, 1264 (2017).
[37] D. R. Smith, Y. Tsai, and S. Larouche, Analysis of a Gradient Index Metamaterial Blazed Diffraction Grating, IEEE Antennas Wireless Propag. Lett. 10, 1605 (2011).

[38] A. M. H. Wong and G. V. Eleftheriades, in 32nd URSI General Assembly and Scientific Symposium (IEEE, Montreal, 2017).

[39] See Supplemental Material at http://link.aps.org/ supplemental/10.1103/PhysRevX.8.011036 for (1) a derivation on the maximum allowable discretization in a metasurface and (2) the normalization procedure for power efficiency analysis for an anomalous reflection metasurface.

[40] A. Hessel, J. Schmoys, and D. Y. Tseng, Bragg-Angle Blazing of Diffraction Gratings, J. Opt. Soc. Am. 65, 380 (1975).

[41] M. Memarian, X. Li, Y. Morimoto, and T. Itoh, Wide-Band/ Angle Blazed Surfaces Using Multiple Coupled Blazing Resonances, Sci. Rep. 7, 42286 (2017).

[42] A. M. H. Wong, P. Christian, and G. V. Eleftheriades, in United States National Committee of URSI National Radio Science Meeting (IEEE, Boulder, 2017).

[43] See Supplemental Material at http://link.aps.org/ supplemental/10.1103/PhysRevX.8.011036 for animations of the waves incident to and reflected from the bipartite Huygens' metasurface.

[44] R. J. Mailloux, Phased Array Antenna Handbook, 2nd ed. (Artech House, Boston, 2005).

[45] J. Huang and J. A. Encinar, Reflectarray Antennas (John Wiley \& Sons, Inc., New York, 2008).

[46] A. Epstein and G. V. Eleftheriades, Floquet-Bloch Analysis of Refracting Huygens Metasurfaces, Phys. Rev. B 90, 235127 (2014).

[47] Y. Ra'di, D. L. Sounas, and A. Alù, Metagratings: Beyond the Limits of Graded Metasurfaces for Wave Front Control, Phys. Rev. Lett. 119, 067404 (2017). 